\title{
A REGULARIZING LEVENBERG-MARQUARDT SCHEME, WITH APPLICATIONS TO INVERSE GROUNDWATER FILTRATION PROBLEMS
}

\author{
MARTIN HANKE*
}

\begin{abstract}
The first part of this paper studies a Levenberg-Marquardt scheme for nonlinear inverse problems where the corresponding Lagrange (or regularization) parameter is chosen from an inexact Newton strategy. While the convergence analysis of standard implementations based on trust region strategies always requires the invertibility of the Fréchet derivative of the nonlinear operator at the exact solution, the new Levenberg-Marquardt scheme is suitable for ill-posed problems as long as the Taylor remainder is of second order in the interpolating metric between the range and domain topologies.

Estimates of this type are established in the second part of the paper for ill-posed parameter identification problems arising in inverse groundwater hydrology. Both, transient and steady state data are investigated. Finally, the numerical performance of the new Levenberg-Marquardt scheme is studied and compared to a usual implementation on a realistic but synthetic 2D model problem from the engineering literature.
\end{abstract}

Key words. Nonlinear ill-posed problems, Levenberg-Marquardt method, Tikhonov regularization, parameter identification, convergence analysis.

AMS subject classifications. 65J15, 65J20, 35R30

1. Introduction. Inverse problems are often solved by approximately minimizing the so-called output least squares functional

$$
\left\|u^{\delta}-F(a)\right\|^{2},
$$

where $F: \mathcal{D}(F) \subset \mathcal{X} \rightarrow \mathcal{Y}$ is a nonlinear differentiable operator between Hilbert spaces $\mathcal{X}$ and $\mathcal{Y} \Gamma$ and $u^{\delta}$ are the given data. In many applications it follows from physical considerations that $u^{\delta}$ is a reasonably close approximation of some ideal $u=F\left(a^{\dagger}\right)$ in the range of $F$ Thence the minimization of (1.1).

The Levenberg-Marquardt method is a variant of the Gauß-Newton iteration for the minimization of (1.1). Given a current approximation $a_{n}$ for $a^{\dagger}$ the nonlinear mapping $F(a)$ in (1.1) is replaced by its linearization around $a_{n}$ prior to the minimization process. If the inverse problem is ill-posed $\Gamma$ however $\Gamma$ neither the original problem of minimizing (1.1) nor its linearized counterpart need to have a solution; even worse if a minimizer does exist Tit can be arbitrarily far off from the true solution $a^{\dagger}$. This is important in many applications where one is interested in properties of $a^{\dagger}$ itself and not in $F\left(a^{\dagger}\right)$.

To overcome this instability one can proceed along several linesTleading to different motivations for essentially the same algorithm (cf.Te.g. TVogel [16]). In the LevenbergMarquardt method a trust region is chosen around $a_{n}$ Гi.e. . a ball of radius $\eta_{n}$ Tand the linearized functional is minimized within this ball. This is easily seen to be equivalent to minimizing

$$
\left\|u^{\delta}-F\left(a_{n}\right)-F^{\prime}\left(a_{n}\right) h\right\|^{2}+\alpha_{n}\|h\|^{2} \longrightarrow \min .
$$

\footnotetext{
* Fachbereich Mathematik, Universität Kaiserslautern, D-67653 Kaiserslautern, Germany. email: hanke@math. uni-karlsruhe.de
} 
for $h=h_{n}$ Twhere $\alpha_{n}$ is the corresponding Lagrange parameter. Then this is repeated with $a_{n+1}=a_{n}+h_{n}$ instead of $a_{n}$ and (possibly) some updated trust region radius $\eta_{n+1}$ until convergence. The difficulty in this approach is an appropriate strategy for choosing $\left\{\eta_{n}\right\}$ Twhich must rely on heuristical considerations. Most standard strategies (cf. Dennis and Schnabel [6] or Nash and Sofer [15]) Tin first placeГdo not cope with the ill-posedness of the problem; they have originally been developed to "globalize" the convergence of the Gauß-Newton iteration for well-posed minimization problems.

On the other handTanother justification for (1.2) is the regularization induced by adding the penalty term $\alpha_{n}\|h\|^{2}$ to the linearized functional. This is equivalent to Tikhonov's method (cf.Te.g.TGroetsch [10]) applied to the linearized problem

$$
F^{\prime}\left(a_{n}\right) h=u^{\delta}-F\left(a_{n}\right) .
$$

Note that for $h_{n}^{\dagger}=a^{\dagger}-a_{n}$ equation (1.3) holds up to the error

$$
\varepsilon_{n}=u^{\delta}-u+R\left(a^{\dagger} ; a_{n}\right)
$$

in the right-hand side $\mathrm{w}$ where $R\left(a^{\dagger} ; a_{n}\right)$ denotes the Taylor remainder for the linearization around $a_{n}$.

At this point it might be better to select $\alpha_{n}$ from a trust region approach for $\varepsilon_{n}$ in $\mathcal{Y}$ rather than some trust region around $a_{n}$ in $\mathcal{X}$. For example $\mathrm{T}$ assume that the right-hand side of (1.3) dominates $\varepsilon_{n}$ Ti.e. $\Gamma\left\|\varepsilon_{n}\right\| \leq \rho\left\|u^{\delta}-F\left(a_{n}\right)\right\|$ for some $\rho<$ 1. The minimal norm solution of (1.3) subject to this constraint on the data fit is characterized by the same minimization problem (1.2) with the difference that $\alpha_{n}$ is now the reciprocal of the corresponding Lagrange parameter.

It will be shown below that this latter choice of $\alpha_{n}$ leads to stable LevenbergMarquardt approximations of $a^{\dagger}$ Tprovided that $F^{\prime}(a)$ is locally bounded and the Taylor remainder $R(\tilde{a} ; a)$ satisfies the inequality

$$
\|R(\tilde{a} ; a)\| \leq C\|\tilde{a}-a\|\|F(\tilde{a})-F(a)\|
$$

for all $\tilde{a}, a$ in a ball $\mathcal{B} \subset \mathcal{D}(F)$ around $a^{\dagger}$ and some fixed $C>0$. This assumption is fulfilled for an important inverse problem in groundwater filtration $\Gamma$ for which numerical examples will be given at the end of the paper.

2. Monotonicity of the Levenberg-Marquardt iterates. Let $\rho$ and $\gamma$ be positive parameters with $\rho<1<\gamma$. Assume that after $n$ iterations $h_{n}^{\dagger}=a^{\dagger}-a_{n}$ satisfies (1.3) up to an error

$$
\left\|u^{\delta}-F\left(a_{n}\right)-F^{\prime}\left(a_{n}\right) h_{n}^{\dagger}\right\| \leq \frac{\rho}{\gamma}\left\|u^{\delta}-F\left(a_{n}\right)\right\| .
$$

Denote by $h_{n, \alpha}$ the minimizer of (1.2) for a given parameter $\alpha_{n}=\alpha$ Tand define the actual Levenberg-Marquardt parameter $\alpha_{n}$ from Morozov's discrepancy principle

$$
\left\|u^{\delta}-F\left(a_{n}\right)-F^{\prime}\left(a_{n}\right) h_{n, \alpha_{n}}\right\|=\rho\left\|u^{\delta}-F\left(a_{n}\right)\right\| .
$$

It is well-known (cf. Groetsch [10Гpp. 44]) that $\alpha_{n}$ is uniquely determined from (2.2). MoreoverTamong all $h \in \mathcal{X}$ with $\left\|u^{\delta}-F\left(a_{n}\right)-F^{\prime}\left(a_{n}\right) h\right\| \leq \rho\left\|u^{\delta}-F\left(a_{n}\right)\right\|$ (including $\left.h=h_{n}^{\dagger}\right) h_{n, \alpha_{n}}$ is the unique element of minimal norm. The following result shows that $a_{n}+h_{n, \alpha_{n}}$ is a better approximation of $a^{\dagger}$ than $a_{n}$. 
Proposition 2.1. Let $0<\rho<1<\gamma$ and assume that (2.1) is fulfilled so that $\alpha_{n}$ can be defined via (2.2). Then, with $v_{n}=\left(F^{\prime}\left(a_{n}\right) F^{\prime}\left(a_{n}\right)^{*}+\alpha_{n} I\right)^{-1}\left(u^{\delta}-F\left(a_{n}\right)\right)$ there holds

$$
\left\|a^{\dagger}-a_{n}\right\|^{2}-\left\|a^{\dagger}-\left(a_{n}+h_{n, \alpha}\right)\right\|^{2}>\frac{2(\gamma-1) \rho}{\gamma}\left\|u^{\delta}-F\left(a_{n}\right)\right\|\left\|v_{n}\right\|,
$$

and also

$$
\left\|a^{\dagger}-a_{n}\right\|^{2}-\left\|a^{\dagger}-\left(a_{n}+h_{n, \alpha}\right)\right\|^{2}>\frac{2(\gamma-1) \rho(1-\rho)}{\gamma\left\|F^{\prime}\left(a_{n}\right)\right\|^{2}}\left\|u^{\delta}-F\left(a_{n}\right)\right\|^{2} .
$$

Proof. For ease of notation let $T=F^{\prime}\left(a_{n}\right) \Gamma \tilde{y}=u^{\delta}-F\left(a_{n}\right)$ and $\Gamma$ as before $\Gamma$ $h_{n}^{\dagger}=a^{\dagger}-a_{n}$. Since for $\alpha>0$

$$
h_{n, \alpha}=T^{*}\left(T T^{*}+\alpha I\right)^{-1} \tilde{y}, \quad \tilde{y}-T h_{n, \alpha}=\alpha\left(T T^{*}+\alpha I\right)^{-1} \tilde{y},
$$

cf. [10] Tit follows that

$$
\begin{aligned}
&\left\|h_{n, \alpha}-h_{n}^{\dagger}\right\|^{2}=\left\|h_{n, \alpha}\right\|^{2}-2\left\langle h_{n, \alpha}, h_{n}^{\dagger}\right\rangle+\left\|h_{n}^{\dagger}\right\|^{2} \\
&=\left\langle\tilde{y}, T T^{*}\left(T T^{*}+\alpha I\right)^{-2} \tilde{y}\right\rangle-2\left\langle\left(T T^{*}+\alpha I\right)^{-1} \tilde{y}, T h_{n}^{\dagger}\right\rangle+\left\|h_{n}^{\dagger}\right\|^{2} \\
&=\left\langle\tilde{y}, T T^{*}\left(T T^{*}+\alpha I\right)^{-2} \tilde{y}\right\rangle-2\left\langle\tilde{y},\left(T T^{*}+\alpha I\right)^{-1} \tilde{y}\right\rangle \\
& \quad+2\left\langle\left(T T^{*}+\alpha I\right)^{-1} \tilde{y}, \tilde{y}-T h_{n}^{\dagger}\right\rangle+\left\|h_{n}^{\dagger}\right\|^{2} \\
&=-\left\langle\tilde{y}, T T^{*}\left(T T^{*}+\alpha I\right)^{-1} \tilde{y}\right\rangle-2 \alpha\left\langle\tilde{y},\left(T T^{*}+\alpha I\right)^{-2} \tilde{y}\right\rangle \\
& \quad+2\left\langle\left(T T^{*}+\alpha I\right)^{-1} \tilde{y}, \tilde{y}-T h_{n}^{\dagger}\right\rangle+\left\|h_{n}^{\dagger}\right\|^{2} .
\end{aligned}
$$

By virtue of (2.1) $\tilde{y}$ does not belong to the orthogonal complement of the range of $T \Gamma$ i.e. $\Gamma T T^{*} \tilde{y} \neq 0$ Tand hence $\Gamma\left\langle\tilde{y}, T T^{*}\left(T T^{*}+\alpha I\right)^{-1} \tilde{y}\right\rangle$ is positive. As a consequence $\Gamma$ this implies that

$$
\begin{aligned}
& \left\|h_{n}^{\dagger}\right\|^{2}-\left\|h_{n, \alpha}-h_{n}^{\dagger}\right\|^{2} \\
& \quad>2 \alpha\left\|\left(T T^{*}+\alpha I\right)^{-1} \tilde{y}\right\|^{2}-2\left\|\left(T T^{*}+\alpha I\right)^{-1} \tilde{y}\right\|\left\|\tilde{y}-T h_{n}^{\dagger}\right\| .
\end{aligned}
$$

Consider now $\alpha=\alpha_{n}$ Ti.e.T the solution of (2.2). In this case the assumptions yield $\left\|\tilde{y}-T h_{n}^{\dagger}\right\| \leq \frac{\rho}{\gamma}\|\tilde{y}\|$ and $\left\|\tilde{y}-T h_{n, \alpha_{n}}\right\|=\rho\|\tilde{y}\|$. In view of (2.5) Tthese two relations can be used in (2.6) to obtain the inequality

$$
\begin{aligned}
\left\|h_{n}^{\dagger}\right\|^{2}-\left\|h_{n, \alpha_{n}}-h_{n}^{\dagger}\right\|^{2} & >2\left\|\left(T T^{*}+\alpha_{n} I\right)^{-1} \tilde{y}\right\|\left(\rho\|\tilde{y}\|-\frac{\rho}{\gamma}\|\tilde{y}\|\right) \\
& =\frac{2(\gamma-1) \rho}{\gamma}\|\tilde{y}\|\left\|\left(T T^{*}+\alpha_{n} I\right)^{-1} \tilde{y}\right\| .
\end{aligned}
$$

Since $\tilde{y}=u^{\delta}-F\left(a_{n}\right)$ and $\left(T T^{*}+\alpha_{n} I\right)^{-1} \tilde{y}=v_{n}$ the first assertion (2.3) follows.

For $\alpha>\frac{\rho}{1-\rho}\|T\|^{2}$ there holds

$$
\frac{\alpha}{\lambda+\alpha}>\rho, \quad 0 \leq \lambda \leq\|T\|^{2},
$$


and henceГcf. (2.5)Г

$$
\left\|\tilde{y}-T h_{n, \alpha}\right\|=\left\|\alpha\left(T T^{*}+\alpha I\right)^{-1} \tilde{y}\right\|>\rho\|\tilde{y}\| \quad \text { for } \alpha>\frac{\rho}{1-\rho}\|T\|^{2} .
$$

This shows that $\alpha_{n} \leq \frac{\rho}{1-\rho}\|T\|^{2}$ Tand since $\tilde{y}=\left(T T^{*}+\alpha I\right) v_{n}$ this implies that

$$
\|\tilde{y}\| \leq\left\|T T^{*}+\alpha I\right\|\left\|v_{n}\right\|=\left(\|T\|^{2}+\alpha\right)\left\|v_{n}\right\| \leq \frac{1}{1-\rho}\|T\|^{2}\left\|v_{n}\right\| .
$$

Inequality (2.4) now follows by using this as a lower bound for $\left\|v_{n}\right\|$ in (2.3).

Since the Levenberg-Marquardt iteration proceeds with

$$
a_{n+1}=a_{n}+h_{n, \alpha_{n}}=a_{n}+F^{\prime}\left(a_{n}\right)^{*} v_{n},
$$

cf. (2.5) $\Gamma v_{n}$ plays an important role in this iterative scheme. On the basis of Proposition 2.1 the following convergence result can now be established.

THEOREM 2.2. Let $0<\rho<1$ and assume that $F^{\prime}(\cdot)$ is locally bounded and that the Taylor remainder of $F$ satisfies (1.5) for some $C>0$. If $u^{\delta}=u=F\left(a^{\dagger}\right)$ and if $a_{0} \in \mathcal{B}$ with $\left\|a_{0}-a^{\dagger}\right\|<\rho / C$ then the Levenberg-Marquardt iteration with $\left\{\alpha_{n}\right\}$ determined from (2.2) converges to a solution of $F(a)=u$ as $n \rightarrow \infty$.

Proof. Define $\gamma=\rho /\left(C\left\|a^{\dagger}-a_{0}\right\|\right)$ which is greater than 1 by assumption. Therefore (1.5) with $\tilde{a}=a^{\dagger}$ and $a=a_{0}$ implies (2.1) Tand hence $\Gamma$

$$
\left\|a^{\dagger}-a_{n+1}\right\|<\left\|a^{\dagger}-a_{n}\right\|
$$

for $n=0$ by virtue of Proposition 2.1. By induction this inequality remains true for all $n$ showing that $\left\|a^{\dagger}-a_{n}\right\|$ is monotonically decreasing during the entire iteration.

It is more difficult to establish the convergence of $\left\{a_{n}\right\}$ to a solution of $F(a)=u$. The proof Thowever Tis the same as the one for Theorem 4.2 in [11] $\Gamma$ and is therefore omitted here.

While Theorem 2.2 shows that the iterates $\left\{a_{n}\right\}$ of the Levenberg-Marquardt iteration converge to a minimizer of (1.1) if the data $u=F\left(a^{\dagger}\right)$ in (1.1) are given exactly the sequence cannot converge if no solution of $F(a)=u^{\delta}$ exists. From linear problems it is well-known (cf. [7]) that the iteration will rather exhibit a semiconvergent behaviour in this case: the iterates seemingly converge in the beginning of the iteration before they eventually turn to diverge. To prevent divergence and to compute stable approximations of $a^{\dagger}$ or some other solution of the unperturbed problem a suitable stopping rule has to be supplied.

For the present version of the Levenberg-Marquardt iteration the discrepancy principle is an appropriate stopping rule for this purpose. Assume that

$$
\left\|u^{\delta}-F\left(a^{\dagger}\right)\right\| \leq \delta,
$$

and to emphasize the dependency on $\delta$ in the remainder of this section let $\left\{a_{n}^{\delta}\right\}$ denote the iterates if $u^{\delta}$ instead of $u$ is used in the iteration. According to the discrepancy principle the iteration is terminated as soon as

$$
\left\|u^{\delta}-F\left(a_{n}^{\delta}\right)\right\| \leq \tau \delta
$$

is fulfilled for the first time with $\tau>1$ another parameter. The following result shows that this stopping rule is well-defined and provides a stable approximation of a solution of $F(a)=u$. 
THEOREM 2.3. Let $0<\rho<1$ and $\tau>1 / \rho$. Assume that $F^{\prime}(\cdot)$ is locally bounded in $\mathcal{D}(F)$ and that the Taylor remainder of $F$ satisfies (1.5). If $\left\|u-u^{\delta}\right\| \leq \delta$ and if $a_{0} \in \mathcal{B}$ is sufficiently close to a solution $a^{\dagger}$ of $F(a)=u$ then the discrepancy principle (2.8) terminates the Levenberg-Marquardt scheme with parameters $\left\{\alpha_{n}\right\}$ from (2.2) after $n(\delta)<\infty$ iterations. Moreover, the corresponding approximations $a_{n(\delta)}^{\delta}$ converge to a solution of $F(a)=u$ as $\delta \rightarrow 0$.

Proof. Let $C$ be the constant in (1.5). At first it will be shown that

$$
\left\|a-a_{n}^{\delta}\right\|<\left\|a-a_{n-1}^{\delta}\right\|, \quad n=1, \ldots, n(\delta),
$$

provided that $a$ is a solution of $F(a)=u$ Tand that an open ball around $a$ of radius $\frac{1}{C}(\rho \tau-1) /(\tau+1)$ including $a_{0}$ belongs to $\mathcal{B}$. In this case it follows from (1.4) and (1.5) that

$$
\begin{aligned}
\| u^{\delta}-F\left(a_{0}\right) & -F^{\prime}\left(a_{0}\right)\left(a-a_{0}\right)\|\leq \delta+C\| a-a_{0}\|\| u-F\left(a_{0}\right) \| \\
& \leq\left(1+C\left\|a-a_{0}\right\|\right) \delta+C\left\|a-a_{0}\right\|\left\|u^{\delta}-F\left(a_{0}\right)\right\| .
\end{aligned}
$$

If $n(\delta)>0$ then $\delta<\left\|u^{\delta}-F\left(a_{0}\right)\right\| / \tau$ Tand hence $\Gamma$

$$
\left\|u^{\delta}-F\left(a_{0}\right)-F^{\prime}\left(a_{0}\right)\left(a-a_{0}\right)\right\| \leq \frac{1+(1+\tau) C\left\|a-a_{0}\right\|}{\tau}\left\|u^{\delta}-F\left(a_{0}\right)\right\| .
$$

This shows that (2.1) holds for $n=0$ with $\gamma=\rho \tau /\left(1+(1+\tau) C\left\|a-a_{0}\right\|\right)$ Twhich is greater than 1 by assumption. Consequently Proposition 2.1 applies and the monotonicity assertion (2.9) follows as in the proof of Theorem 2.2.

Now assume that $a_{0}$ is so close to $a^{\dagger}$ that (2.9) holds for $a=a^{\dagger}$. Then Ttaking the sum of $(2.4)$ for $n=0, \ldots, n(\delta)-1$ one obtains

$$
n(\delta) \tau^{2} \delta^{2} \leq \sum_{n=0}^{n(\delta)-1}\left\|u^{\delta}-F\left(a_{n}^{\delta}\right)\right\|^{2} \leq \frac{\gamma c^{2}}{2 \rho(1-\rho)(\gamma-1)}\left\|a^{\dagger}-a_{0}\right\|^{2}<\infty,
$$

where $c$ is a uniform bound for $\left\|F^{\prime}\left(a_{n}^{\delta}\right)\right\|$ in $\mathcal{B}$. This shows that $n(\delta)$ is a finite number.

Next $\Gamma$ consider $a_{n(\delta)}^{\delta}$ as $\delta \rightarrow 0$. By continuity as $\delta \rightarrow 0$ एwhere $a_{n}$ is the $n$th Levenberg-Marquardt iterate with exact right-hand side $u$. Furthermore $\Gamma$ since $\left\|u^{\delta}-F\left(a_{n}^{\delta}\right)\right\| \leq \tau \delta$ by definition of $n=n(\delta)$ there must hold $F\left(a_{n}\right)=u$ in the limit $\delta \rightarrow 0$. Consequently $\Gamma a_{n(\delta)}^{\delta}$ converges to the solution $a_{n}$ of $F(a)=u$ in this first case that $n(\delta)=n$ for all $\delta>0$.

Finally Tassume that $n(\delta) \rightarrow \infty$ as $\delta \rightarrow 0$ Tand denote by $a$ the limit of $\left\{a_{n}\right\}$ which exists by virtue of Theorem 2.2. Since

$$
\left\|a-a^{\dagger}\right\|=\lim _{n \rightarrow \infty}\left\|a_{n}-a^{\dagger}\right\| \leq\left\|a_{0}-a^{\dagger}\right\|,
$$

cf. (2.9) Гit follows that

$$
\left\|a-a_{0}\right\| \leq\left\|a-a^{\dagger}\right\|+\left\|a^{\dagger}-a_{0}\right\| \leq 2\left\|a^{\dagger}-a_{0}\right\| \leq \frac{\rho \tau-1}{C(\tau+1)},
$$

provided that $a_{0}$ is sufficiently close to $a^{\dagger}$. Therefore (2.9) applies with $a$ being the limit of $\left\{a_{n}\right\}$. Given $\varepsilon>0$ let $m(\varepsilon)$ be such that $\left\|a-a_{m}\right\|<\varepsilon / 2$ for $m>m(\varepsilon)$ and let $\delta(\varepsilon)$ be so small that $n(\delta)>m(\varepsilon)$ for $\delta<\delta(\varepsilon)$; then it follows from $(2.9)$ that

$$
\left\|a-a_{n(\delta)}^{\delta}\right\|<\left\|a-a_{m}^{\delta}\right\| \leq \varepsilon / 2+\left\|a_{m}-a_{m}^{\delta}\right\|
$$


for $m=m(\varepsilon)$ and all $\delta<\delta(\varepsilon)$. Again by continuity it follows that $\left\|a_{m}-a_{m}^{\delta}\right\|<\varepsilon / 2$ and hence $\left\|a-a_{n(\delta)}^{\delta}\right\|<\varepsilon$ for $\delta$ sufficiently small. This proves that $a_{n(\delta)}^{\delta} \rightarrow a$ as $\delta \rightarrow 0$ in the case where $n(\delta) \rightarrow \infty$.

The general case now follows by considering appropriate subsequences of $n(\delta)$ if necessary. $\square$

It remains to comment on the rate of convergenceTi.e. Ton the magnitude of $n(\delta)$. According to the parameter choice rule (2.2) there holds

$$
\left\|u^{\delta}-F\left(a_{n}^{\delta}\right)-F^{\prime}\left(a_{n}^{\delta}\right)\left(a_{n+1}^{\delta}-a_{n}^{\delta}\right)\right\|=\rho\left\|u^{\delta}-F\left(a_{n}^{\delta}\right)\right\|, \quad n=0,1, \ldots, n(\delta)-1 .
$$

Using the triangle inequality and assumption (1.5) it follows that

$$
\begin{aligned}
\rho\left\|u^{\delta}-F\left(a_{n}^{\delta}\right)\right\| \geq & \left\|u^{\delta}-F\left(a_{n+1}^{\delta}\right)\right\|-\left\|F\left(a_{n+1}^{\delta}\right)-F\left(a_{n}^{\delta}\right)-F^{\prime}\left(a_{n}^{\delta}\right)\left(a_{n+1}^{\delta}-a_{n}^{\delta}\right)\right\| \\
\geq & \left\|u^{\delta}-F\left(a_{n+1}^{\delta}\right)\right\|-C\left\|a_{n+1}^{\delta}-a_{n}^{\delta}\right\|\left\|F\left(a_{n+1}^{\delta}\right)-F\left(a_{n}^{\delta}\right)\right\| \\
\geq & \left(1-C\left\|a_{n+1}^{\delta}-a_{n}^{\delta}\right\|\right)\left\|u^{\delta}-F\left(a_{n+1}^{\delta}\right)\right\| \\
& -C\left\|a_{n+1}^{\delta}-a_{n}^{\delta}\right\|\left\|u^{\delta}-F\left(a_{n}^{\delta}\right)\right\|,
\end{aligned}
$$

and hence $\Gamma$

$$
\left\|u^{\delta}-F\left(a_{n+1}^{\delta}\right)\right\| \leq \frac{\rho+C\left\|a_{n+1}^{\delta}-a_{n}^{\delta}\right\|}{1-C\left\|a_{n+1}^{\delta}-a_{n}^{\delta}\right\|}\left\|u^{\delta}-F\left(a_{n}^{\delta}\right)\right\| .
$$

The fraction on the right-hand side is below 1 for $\delta$ sufficiently small and $n$ sufficiently large which yields the following result.

THEOREM 2.4. Under the assumptions of Theorem 2.3 the stopping index $n(\delta)$ grows like $n(\delta)=O(|\log \delta|)$ as $\delta \rightarrow 0$.

It can also be seen from (2.10) that the asymptotic convergence factor for the decay rate of the residuals will approach $\rho$ as $\delta \rightarrow 0$.

Remark. Theorems 2.2 and 2.3 actually hold for a considerably larger class of strategies for choosing the Levenberg-Marquardt parameter $\alpha_{n}$ in (1.2). Roughly speaking $\Gamma$ any continuous parameter choice rule (i.e. $\Gamma$ a rule for which $\alpha_{n}$ depends continuously on $u^{\delta}$ ) with $\alpha_{n}$ greater than the solution of $(2.2)$ will do. This is so because for those parameters (2.2) can be replaced by

$$
\left\|u^{\delta}-F\left(a_{n}^{\delta}\right)-F^{\prime}\left(a_{n}^{\delta}\right) h_{n, \alpha_{n}}\right\| \geq \rho\left\|u^{\delta}-F\left(a_{n}^{\delta}\right)\right\|,
$$

cf. [10ГThm. 3.3.1] Twhich suffices for the proof of Proposition 2.1.

For example $\Gamma$ if $\tau>2$ then alternative choices include $\alpha_{n}=\left\|F^{\prime}\left(a_{n}^{\delta}\right)\right\|^{2}$ in view of (2.7) Гor $\alpha_{n}=\alpha$ Ta constant greater than $\left\|F^{\prime}(a)\right\|^{2}$ for all $a \in \mathcal{B}$. A particularly attractive choice for $\alpha_{n}$ is a strategy developed by EnglГ Gfrerer and Raus (cf. [7Г Sect. 4.4]) for choosing the regularization parameter in Tikhonov regularization for linear problems. This parameter choice rule which determines $\alpha_{n}$ from

$$
\begin{gathered}
\left\langle r_{\alpha}, \alpha\left(F^{\prime}\left(a_{n}^{\delta}\right) F^{\prime}\left(a_{n}^{\delta}\right)^{*}+\alpha I\right)^{-1} r_{\alpha}\right\rangle=\rho^{2}\left\|u^{\delta}-F\left(a_{n}^{\delta}\right)\right\|^{2}, \\
\text { with } r_{\alpha}=u^{\delta}-F\left(a_{n}^{\delta}\right)-F^{\prime}\left(a_{n}^{\delta}\right) h_{n, \alpha},
\end{gathered}
$$

has the advantage that under assumption (2.1) the corresponding error is always smaller than for any greater parameter $\alpha$ (cf. [7TProp. 5.12]). The solution $\alpha_{n}$ of (2.11) is somewhat greater than the solution of $(2.2)$ Tbut always less than $\alpha=c\left\|F^{\prime}\left(a_{n}^{\delta}\right)\right\|^{2}$ for some $c$ depending only on $\rho$. 
3. An inverse problem in groundwater hydrology. Let $\Omega$ be a bounded domain in $\mathbb{R}^{N} \Gamma N \geq 2 \Gamma$ with smooth $\left(C^{2}\right)$ boundary $\Gamma \Gamma$ and consider the boundary value problem

$$
\begin{gathered}
u_{t}-\operatorname{div}(a \operatorname{grad} u)=f \quad \text { in } \Omega, \\
u=\varphi \quad \text { on } \Gamma,
\end{gathered}
$$

where $f$ and $\varphi$ are functions of time $t$ with values in $\mathcal{H}^{-1}(\Omega)$ and $\mathcal{H}^{1 / 2}(\Gamma)$ Trespectively. This differential equation is used as a model for groundwater flow $\Gamma$ where $a$ is the diffusivity of the sediment $\Gamma u$ the piezometric head $\Gamma$ and $f$ represents water sources and sinks. Further applications of (3.1) are discussed by Banks and Kunisch [2].

Given Cauchy data $u_{0} \in \mathcal{L}^{2}(\Omega)$ the direct problem associated with (3.1) consists in finding a solution $u$ of (3.1) in a time interval $\mathcal{T}=[0, T]$ with $u(0)=u_{0}$ for given functions $a \Gamma f \Gamma$ and $\varphi$. Under mild assumptions on $a \Gamma$ namely $a \in \mathcal{L}^{\infty}(\Omega)$ with $a(x) \geq \underline{a}>0$ for all $x \in \Omega(3.1)$ has a weak solution $u$ with values in $H^{1}(\Omega) \Gamma c f . \Gamma$ e.g. $\Gamma$ Dautray and Lions [5]. Let $F: a \mapsto u$ denote this parameter-to-solution mapping.

The inverse problem is aiming for the material coefficient $a$ in order to explore the internal structure of the aquifer $\Omega$. To this end $\Gamma$ wells are drilled to measure $u$ in $\Omega(f$ and $\varphi$ are presumably known) Tand then the nonlinear problem $F(a)=u$ is solved for $a$. HenceTthis corresponds to the setting of the introduction. In the sequel it shall be assumed that distributed data for $u$ are given in $\mathcal{L}^{2}(\mathcal{T} \times \Omega)$. If only discrete measurements are available (e.g. T the values of $u$ at the wells) this may call for an interpolation of these measurements prior to reconstruction $\Gamma$ cf. Section 4.2. In this setting $\Gamma$ i.e. with $\mathcal{X} \subset \mathcal{L}^{\infty}$ and $\mathcal{Y}=\mathcal{L}^{2}(\mathcal{T} \times \Omega)$ the inverse problem is known to be ill-posed $\Gamma$ cf.Te.g.TKravaris and Seinfeld [13] or [2ГSect. 4.2].

In practical applications both the steady state of (3.1) (where $f$ and $\varphi$ Tand hence $u$ do not depend on time) as well as the overdetermined transient case are of interest. In either case the validity of the basic assumption (1.5) for the results of Section 2 hinges on the $\mathcal{H}^{2}$ regularity of the elliptic operator

$$
A u=-\operatorname{div}(a \operatorname{grad} u),
$$

defined for the moment on $\mathcal{H}_{0}^{1}(\Omega)$ in the usual weak sense. Here $\mathrm{A}$ is called $\mathcal{H}^{2}$ regular if $\|u\|_{\mathcal{H}^{2}(\Omega)}$ can be bounded by $\|A u\|_{\mathcal{L}^{2}(\Omega)}$ for any function $u \in \mathcal{H}^{2}(\Omega) \cap \mathcal{H}_{0}^{1}(\Omega)$. Note that $\mathcal{H}^{2}$ regularity has also been employed as an essential ingredient in [2ГChapter 6 ] (see also [4]) for a convergence analysis of certain constrained projection methods for reconstructing $a^{\dagger}$.

$\mathcal{H}^{2}$ regularity imposes a certain smoothness on the parameter function a (cf. Ladyzhenskaya and Ural'tseva [14] or Grisvard [9])Гe.g. Г $a \in \mathcal{W}^{1, p}(\Omega)$ with $p>N$. Here $\Gamma \mathcal{W}^{1, p}(\Omega)$ is the usual Sobolev space of functions with weak derivatives in $\mathcal{L}^{p}(\Omega) \Gamma$ cf. Adams [1]. The restricition $a \in \mathcal{W}^{1, p}(\Omega)$ will also serve as a basic assumption for the analysis to come; it implies that $A$ is an isomorphism between $V:=\mathcal{H}^{2}(\Omega) \cap \mathcal{H}_{0}^{1}(\Omega)$ and $\mathcal{L}^{2}(\Omega) \Gamma$ cf. [14Г p. 184]. Denote by $V^{\prime}$ the dual space of $V$ with respect to the bilinear form

$$
\langle v, w\rangle=\int_{\Omega} v(x) w(x) d x,
$$

and identify the corresponding dual space of $\mathcal{L}^{2}(\Omega)$ with itself. Then the associated dual operator $A^{\prime}: \mathcal{L}^{2}(\Omega) \rightarrow V^{\prime}$ of $A$ is an isomorphic extension of $A$ to $\mathcal{L}^{2}(\Omega)$ because 
$A$ is symmetric when considered as a densely defined operator in $\mathcal{L}^{2}(\Omega)$. The same symbol $A$ will again be used for this extension to all of $\mathcal{L}^{2}(\Omega)$ further on.

On the other hand $\Gamma$ for a not necessarily positive function $h \in \mathcal{W}^{1, p}(\Omega)$ with $p>N$ and associated operator

$$
H: \mathcal{H}^{2}(\Omega) \rightarrow \mathcal{L}^{2}(\Omega), \quad H: u \mapsto \operatorname{div}(h \operatorname{grad} u),
$$

one has

$$
\|H\|_{\mathcal{L}^{2}(\Omega) \leftarrow \mathcal{H}^{2}(\Omega)} \leq c\|h\|_{\mathcal{W}^{1, p}(\Omega)},
$$

and the constant $c$ therein depends only on $\Omega$. To see this $\Gamma$ let $u \in C^{\infty}(\bar{\Omega})$ and consider

$$
\begin{aligned}
\|H u\|_{\mathcal{L}^{2}(\Omega)} & =\|h \Delta u+\operatorname{grad} h \cdot \operatorname{grad} u\|_{\mathcal{L}^{2}(\Omega)} \\
& \leq\|h\|_{\mathcal{L}^{\infty}(\Omega)}\|\Delta u\|_{\mathcal{L}^{2}(\Omega)}+\|\operatorname{grad} h \cdot \operatorname{grad} u\|_{\mathcal{L}^{2}(\Omega)} .
\end{aligned}
$$

Using the Hölder inequality the second member of the right-hand side can be estimated from above by $c\|h\|_{\mathcal{W}^{1, p}(\Omega)}\|u\|_{\mathcal{W}^{1, q(\Omega)}}$ with $q=2 p /(p-2)$. Now (3.4) follows because $\mathcal{W}^{1, p}(\Omega)$ embeds continuously into $\mathcal{L}^{\infty}(\Omega)$ and $\mathcal{H}^{2}(\Omega)$ embeds continuously into $\mathcal{W}^{1, q}(\Omega)$ as $p>N$ (cf. [1]).

$H$ extends to an operator $H: \mathcal{H}^{1}(\Omega) \rightarrow V^{\prime}$ in the usual weak sense. The following estimate will be useful below: given $u \in \mathcal{H}^{1}(\Omega)$ and $v \in V$ it follows through integration by parts ( $n$ denotes the outward normal to $\Omega$ on $\Gamma$ ) that

$$
\begin{aligned}
\langle H u, v\rangle & =\int_{\Omega} u \operatorname{div}(h \operatorname{grad} v) d x-\int_{\Gamma} h u \frac{\partial v}{\partial n} d s \\
& \leq\|u\|_{\mathcal{L}^{2}(\Omega)}\|H v\|_{\mathcal{L}^{2}(\Omega)}+\|h u\|_{\mathcal{H}^{-1 / 2}(\Gamma)}\|v\|_{V} \\
& \leq\left(\|u\|_{\mathcal{L}^{2}(\Omega)}\|H\|_{\mathcal{L}^{2}(\Omega) \leftarrow \mathcal{H}^{2}(\Omega)}+\|h\|_{\mathcal{L}^{\infty}(\Omega)}\|u\|_{\mathcal{H}^{-1 / 2}(\Gamma)}\right)\|v\|_{V} .
\end{aligned}
$$

Together with (3.4) this implies that

$$
\|H u\|_{V^{\prime}} \leq c\|h\|_{\mathcal{W}^{1, p}(\Omega)}\left(\|u\|_{\mathcal{L}^{2}(\Omega)}+\|u\|_{\mathcal{H}^{-1 / 2}(\Gamma)}\right) .
$$

3.1. The steady state case. Assume for the time being that $f$ and $\varphi$ are constant in time. Let $a, \tilde{a} \in \mathcal{W}^{1, p}(\Omega)$ be bounded from below by some $\underline{a}>0$ Tand let $A$ and $\tilde{A}$ be the associated operators (3.2). Furthermore be the solutions (which are now functions of space only) of the respective boundary value problems (3.1).

It is instructive to evaluate $A u$ as $u=F(a) \notin V$ Tand hence $A$ here really denotes the extended operator. It turns out that

$$
A u=f-g,
$$

where $g \in V^{\prime}$ is the representation of the continuous linear functional

$$
\langle g, v\rangle=\int_{\Gamma} a \varphi \frac{\partial v}{\partial n} d s, \quad v \in V .
$$


Considered as a mapping from $\mathcal{W}^{1, p}(\Omega)$ to $\mathcal{L}^{2}(\Omega)$ the operator $F$ is differentiable: $u^{\prime}=F^{\prime}(a) h$ is given as the (weak) solution of the boundary value problem

$$
\begin{gathered}
-\operatorname{div}\left(a \operatorname{grad} u^{\prime}\right)=\operatorname{div}(h \operatorname{grad} u) \quad \text { in } \Omega, \\
u^{\prime}=0 \quad \text { on } \Gamma,
\end{gathered}
$$

or rather $\Gamma$

$$
A u^{\prime}=H F(a)
$$

with $A$ and $H$ as above. This formula is well-known in the literature; moreover Tcf. Ito and Kunisch [12] Tthe derivative considered as an operator $F^{\prime}(a): \mathcal{L}^{\infty}(\Omega) \rightarrow \mathcal{L}^{2}(\Omega)$ is a compact operator. This means that not only the nonlinear inverse problem but also the linearized problem (1.3) is ill-posed in general.

Theorem 3.1. Let $\Omega \subset \mathbb{R}^{N}$ be bounded with $C^{2}$ boundary $\Gamma$, and $a, \tilde{a} \in \mathcal{W}^{1, p}(\Omega)$ with $p>N$ be greater than $\underline{a}>0$ in $\Omega$. Then $F^{\prime}(\cdot)$ is uniformly bounded in a $\mathcal{W}^{1, p}(\Omega)$ neighborhood of $a$ and

$$
\left\|F(\tilde{a})-F(a)-F^{\prime}(a)(\tilde{a}-a)\right\|_{\mathcal{L}^{2}(\Omega)} \leq C\|\tilde{a}-a\|_{\mathcal{W}^{1, p}(\Omega)}\|F(\tilde{a})-F(a)\|_{\mathcal{L}^{2}(\Omega)},
$$

where the constants only depend on $\Omega, \underline{a}$ and on the $\mathcal{W}^{1, p}(\Omega)$ norm of $a$.

Proof. Since $A$ of $(3.2)$ is an isomorphism from $\mathcal{L}^{2}(\Omega)$ to $V^{\prime}$ it follows from (3.7) that

$$
F^{\prime}(a) h=A^{-1} H F(a)
$$

and henceГby $(3.5) \Gamma$

$$
\begin{aligned}
\left\|F^{\prime}(a) h\right\|_{\mathcal{L}^{2}(\Omega)} & \leq\left\|A^{-1}\right\|_{\mathcal{L}^{2}(\Omega) \leftarrow V^{\prime}}\|H F(a)\|_{V^{\prime}} \\
& \leq c\|h\|_{\mathcal{W}^{1, p}(\Omega)}\left(\|F(a)\|_{\mathcal{L}^{2}(\Omega)}+\|\varphi\|_{\mathcal{H}^{-1 / 2}(\Gamma)}\right)
\end{aligned}
$$

where the constant $c$ depends only on $\Omega \Gamma \underline{a} \Gamma$ and $\|a\|_{\mathcal{W}^{1, p}(\Omega)} \Gamma \mathrm{cf}$. [14 $\Gamma$ p. 189]. Furthermore $\Gamma$ by $(3.6) \Gamma\|F(a)\|_{\mathcal{L}^{2}(\Omega)}$ depends on the same quantities as before $\Gamma$ as well as on $\|f\|_{V^{\prime}}$ and on $\|\varphi\|_{\mathcal{H}^{-1 / 2}(\Gamma)}$. As a consequence $\Gamma\left\|F^{\prime}(\cdot)\right\|$ is uniformly bounded in a $\mathcal{W}^{1, p}(\Omega)$-neighborhood of $a$.

Now let $u=F(a) \Gamma \tilde{u}=F(\tilde{a}) \Gamma$ and $u^{\prime}=F^{\prime}(a) h$ with $h=\tilde{a}-a$. It will be useful for the sequel to evaluate $A \tilde{u}$ : taking an arbitrary $v \in V$ and integrating by parts it follows from the variational definition of $\tilde{u}$ that

$$
\begin{aligned}
\langle A v, \tilde{u}\rangle & =-\int_{\Gamma} a \varphi \frac{\partial v}{\partial n} d s+\int_{\Omega} a \operatorname{grad} \tilde{u} \cdot \operatorname{grad} v d x \\
& =-\langle g, v\rangle+\int_{\Omega}(a-\tilde{a}) \operatorname{grad} \tilde{u} \cdot \operatorname{grad} v d x+\langle f, v\rangle \\
& =\langle f-g+H \tilde{u}, v\rangle,
\end{aligned}
$$

and henceГ $A \tilde{u}=f-g+H \tilde{u}$. Therefore for

$$
w=\tilde{u}-u-u^{\prime}=F(\tilde{a})-F(a)-F^{\prime}(a)(\tilde{a}-a)
$$


it follows from (3.6) and (3.7) that

$$
A w=A \tilde{u}-A u-A u^{\prime}=f-g+H \tilde{u}-(f-g)-H u=H(\tilde{u}-u) .
$$

Together with (3.5) this yields

$$
\|w\|_{\mathcal{L}^{2}(\Omega)} \leq\left\|A^{-1}\right\|_{\mathcal{L}^{2}(\Omega) \leftarrow V^{\prime}}\|H(\tilde{u}-u)\|_{V^{\prime}} \leq c\|h\|_{\mathcal{W}^{1, p}(\Omega)}\|\tilde{u}-u\|_{\mathcal{L}^{2}(\Omega)},
$$

where again $\Gamma c$ only depends on $\Omega \Gamma \underline{a}$ and on $\|a\|_{\mathcal{W}^{1, p}(\Omega)}$. $\square$

For a 1D version of Theorem 3.1 (with $a$ and $\tilde{a}$ in $\mathcal{H}^{1}(\Omega)$ ) cf. [7ГEx. 11.1].

Since $\mathcal{W}^{1, p}(\Omega)$ is no Hilbert space it is no suitable choice for $\mathcal{X}$ in the setting of Sections 1 and 2. On the other hand $\Gamma$ when $N \leq 3$ then $\mathcal{H}^{2}(\Omega)$ can be continuously embedded into $\mathcal{W}^{1, p}(\Omega)$ for appropriate $p>N$ :

Corolla RY 3.2. Assume that $N=2$ or $N=3$ and that $\Omega$ is as in Theorem 3.1. If $a, \tilde{a} \in \mathcal{H}^{2}(\Omega)$ are bounded in $\Omega$ from below by some $\underline{a}>0$ then

$$
\left\|F(\tilde{a})-F(a)-F^{\prime}(a)(\tilde{a}-a)\right\|_{\mathcal{L}^{2}(\Omega)} \leq C\|\tilde{a}-a\|_{\mathcal{H}^{2}(\Omega)}\|F(\tilde{a})-F(a)\|_{\mathcal{L}^{2}(\Omega)} .
$$

Proof. NoteГcf. [1] Tthat $\mathcal{H}^{2}(\Omega)$ is continuously embedded in $\mathcal{W}^{1, p}(\Omega)$ when $p \geq 2$ and $(N-2) p \leq 2 N$. Thus for $N=2$ and $N=3$ Tevery $a \in \mathcal{H}^{2}(\Omega)$ is embedded in $\mathcal{W}^{1,4}(\Omega)$ Ti.e. $\Gamma|| a\left\|_{\mathcal{W}^{1,4}(\Omega)} \leq c\right\| a \|_{\mathcal{H}^{2}(\Omega)}$ for some fixed $c>0$. Thus the assertion follows from Theorem 3.1 with $p=4$.

In higher dimensional spaces $a \in \mathcal{H}^{2}(\Omega)$ does not suffice in Corollary 3.2. The reason is twofold. First $\Gamma$ the argument in the proof fails for higher dimensions. Secondly the analysis in Section 2 requires that a whole ball in $\mathcal{X}$ around $a^{\dagger}$ contains only strictly positive functions. Consequently $\mathcal{X}$ has to be a subspace of bounded functions.

Remark. Note that in the proof of Theorem 3.1 the functions $u, \tilde{u}$ Tand $w$ need only belong to $\mathcal{L}^{2}(\Omega)$. Therefore the same result holds under considerably weaker assumptions on $f$ and $g$; in particular $\Gamma f \in V^{\prime}$ would be sufficient. This may be of practical importance because it allows the use of delta distributions for $f$ (e.g. Tpoint injections at the wells) provided that $N \leq 3$. i.e. $\mathrm{T}$

3.2. The transient case. When $f$ and $\varphi$ are functions of time $t \Gamma 0 \leq t \leq T \Gamma$

$$
f \in \mathcal{L}^{2}\left(0, T ; \mathcal{H}^{-1}(\Omega)\right), \quad \varphi \in \mathcal{L}^{2}\left(0, T ; \mathcal{H}^{1 / 2}(\Gamma)\right),
$$

and the initial data $u_{0}$ belong to $\mathcal{H}^{1}(\Omega)$ then $(3.1)$ has a unique solution

$$
u=F(a) \in \mathcal{L}^{2}\left(0, T ; \mathcal{H}^{1}(\Omega)\right)
$$

The formal derivative of $F$ with respect to $a$ has a similar form as above $\Gamma$ i.e. $\Gamma u^{\prime}=$ $F^{\prime}(a) h$ solves the Cauchy-Dirichlet problem

$$
\begin{gathered}
u^{\prime}(0)=0, \quad L u^{\prime} \equiv u_{t}^{\prime}-\operatorname{div}\left(a \operatorname{grad} u^{\prime}\right)=H u \quad \text { in } \Omega, \\
u^{\prime}=0 \quad \text { on } \Gamma,
\end{gathered}
$$

where $H$ is given by (3.3) and $u=F(a)$ is the solution of (3.1) with initial data $u(0)=u_{0}$. 
TheOrem 3.3. Let $\Omega \subset \mathbb{R}^{N}$ be bounded with $C^{2}$ boundary $\Gamma$, and $a, \tilde{a} \in \mathcal{W}^{1, p}(\Omega)$ with $p>N$ be greater than $\underline{a}>0$ in $\Omega$. Then $F^{\prime}(\cdot)$ is locally bounded in a $\mathcal{W}^{1, p}(\Omega)$ neighborhood of a and

$\left\|F(\tilde{a})-F(a)-F^{\prime}(a)(\tilde{a}-a)\right\|_{\mathcal{L}^{2}\left(0, T ; \mathcal{L}^{2}(\Omega)\right)} \leq C\|\tilde{a}-a\|_{\mathcal{W}^{1, p}(\Omega)}\|F(\tilde{a})-F(a)\|_{\mathcal{L}^{2}\left(0, T ; \mathcal{L}^{2}(\Omega)\right)}$, where $c$ depends only on $\Omega$, on a and on the $\mathcal{W}^{1, p}(\Omega)$ norm of a. Corollary 3.2 applies accordingly.

Proof. By the regularity result in [5Г p. 116] the dual operator $L^{\prime}$ of $L$ (with respect to $\mathcal{L}^{2}\left(0, T ; \mathcal{L}^{2}(\Omega)\right)$ given by

$$
L^{\prime} v=-v_{t}-\operatorname{div}(a \operatorname{grad} v)
$$

for $v \in \mathcal{L}^{2}\left(0, T ; \mathcal{H}_{0}^{1}(\Omega)\right.$ ) with $v(T)=0$ (which corresponds to an evolution operator backwards in time from $T$ to 0$)$ is bounded from below as operator from $\mathcal{L}^{2}(0, T ; \mathcal{D}(A))$ to $\mathcal{L}^{2}\left(0, T ; \mathcal{L}^{2}(\Omega)\right)$. Under the assumptions of the theorem $\Gamma$

$$
\mathcal{D}(A) \equiv\left\{u \in \mathcal{H}_{0}^{1}(\Omega) \mid A u \in \mathcal{L}^{2}(\Omega)\right\}=V,
$$

and hence $L$ is bounded from below as a mapping

$$
L: \mathcal{L}^{2}\left(0, T ; \mathcal{L}^{2}(\Omega)\right) \rightarrow \mathcal{L}^{2}\left(0, T ; V^{\prime}\right) .
$$

Consequently it follows from (3.8) and (3.5) that for certain $c>0$

$$
\begin{aligned}
\left\|F^{\prime}(a) h\right\|_{\mathcal{L}^{2}\left(0, T ; \mathcal{L}^{2}(\Omega)\right)}^{2} & \leq c \int_{0}^{T}\|H F(a)\|_{V^{\prime}}^{2} d t \\
& \leq c \int_{0}^{T}\|h\|_{\mathcal{W}^{1, p}(\Omega)}^{2}\left(\|F(a)\|_{\mathcal{L}^{2}(\Omega)}^{2}+\|\varphi\|_{\mathcal{H}^{-1 / 2}(\Gamma)}^{2}\right) d t \\
& \leq c\|h\|_{\mathcal{W}^{1, p}(\Omega)}^{2}\left(\|F(a)\|_{\mathcal{L}^{2}\left(0, T ; \mathcal{L}^{2}(\Omega)\right)}^{2}+\|\varphi\|_{\mathcal{L}^{2}\left(0, T ; \mathcal{H}^{-1 / 2}(\Gamma)\right)}^{2}\right) .
\end{aligned}
$$

The boundedness of the derivative now follows similar to the proof of Theorem 3.1.

Furthermore $\Gamma$ if $h=\tilde{a}-a \Gamma u=F(a) \Gamma \tilde{u}=F(\tilde{a}) \Gamma$ and $u^{\prime}$ denotes the solution of $(3.8) \Gamma$ then similar computations as in the proof of Theorem 3.1 establish that $w=\tilde{u}-u-u^{\prime}$ is a solution of

$$
w(0)=0, \quad L w=H(\tilde{u}-u),\left.\quad w\right|_{\Gamma}=0,
$$

and the same inequality chain as before yields

$$
\|w\|_{\mathcal{L}^{2}\left(0, T ; \mathcal{L}^{2}(\Omega)\right)} \leq c\|h\|_{\mathcal{W}^{1, p}(\Omega)}\|\tilde{u}-u\|_{\mathcal{L}^{2}\left(0, T ; \mathcal{L}^{2}(\Omega)\right)}
$$

as was to be shown.

Remark. Once again $\Gamma$ the crucial role of the $\mathcal{H}^{2}$ regularity of the differential operator $A$ of (3.2) for the theorems in this section should be stressed. Whenever $A$ is $\mathcal{H}^{2}$ regular under modified assumptions on $\Omega$ analogous results can be established. In particularTsimilar results follow for convex domains from the regularity results in [9]. 


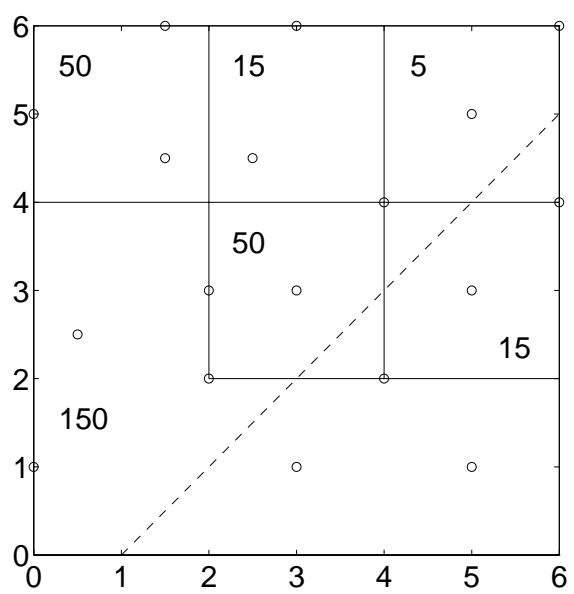

FIG. 4.1. Phantom $a^{\dagger}$ and the locations of the wells

4. Numerical results. The theoretical results in this paper establish that the Levenberg-Marquardt scheme with the parameters $\left\{\alpha_{n}\right\}$ determined from (2.2) can be applied to the above inverse problems from groundwater hydrology. The algorithm has been tested on synthetic data suggested by Carrera and Neuman [3] as a realistic test case for inverse groundwater filtration problems.

The exact diffusivity coefficient $a^{\dagger}$ of this model problem is piecewise constant with significant discontinuities; hence $A$ of (3.2) lacks $\mathcal{H}^{2}$ regularity and the theoretical analysis of this paper does not apply to this particular example. Nevertheless 5 the numerical results are extremely promising and indicate the usefulness of the method for a larger class of problems. This does not mean $\Gamma$ however $\Gamma$ that this algorithm is recommended as the method of choice for this problem. There are a number of alternative approaches $\Gamma$ cf. $\Gamma$ e.g. $\Gamma$ the survey of Yeh [17] and the references in [2Г 3]. More recent contributions with up to date references can be found in the proceedings of the 1994 GAMM-SIAM conference on diffusion processes [8].

The synthetic data from [3] correspond to a square aquifer $(0 \leq \xi, \eta \leq 6)$ with six different transmissivity zones with constant values of $a^{\dagger}$ Tranging from 5 to 150 as displayed in Figure 4.1. The circles in this figure mark the locations of the eighteen wells where head measurements are to be taken; the dashed line will be referred to later on. The piezometric head $u$ solves the differential equation (3.1) with mixed Neumann-Dirichlet boundary data on ГГnamely

$$
\begin{aligned}
u(\xi, 0) & =100, & u_{\xi}(6, \eta) & =0, \\
\left(a u_{\xi}\right)(0, \eta) & =-500, & & u_{\eta}(\xi, 6)=0 .
\end{aligned}
$$

The right-hand side $f$ of (3.1) is given by

$$
f(\xi, \eta)=\left\{\begin{aligned}
0, & 0<\eta<4, \\
137, & 4<\eta<5, \\
274, & 5<\eta<6 .
\end{aligned}\right.
$$

Carrera and Neuman also suggest a transient test case corresponding to a pumping of two out of the eighteen wells. For the ease of computation and to simplify the display of computational outputTnumerical results will only be presented for the steady state case. 


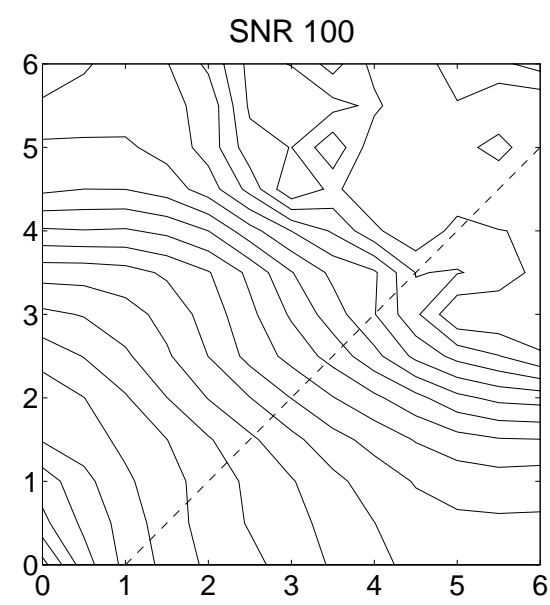

FIG. 4.2. Contour plots of the two reconstructions

The implementation of this example uses a refinement of the triangularization in [3] of the aquifer area with 288 triangles and 169 grid points. The exact diffusivity coefficient $a^{\dagger}$ is used to compute approximate head data with the finite element method; random noise is added to all simulated data. Although the same triangularization is used for the inverse problem $\Gamma$ approximate diffusivity parameters are taken from the subspace of piecewise linear functions. As a consequence the exact diffusivity coefficent does not belong to this trial space; in fact $\Gamma$ the best approximation of $a^{\dagger}$ from this subspace has a relative error of approximately 0.1628 (measured in $\mathcal{L}^{2}(\Omega)$ ).

In this implementation $F$ is considered as a mapping

$$
F: \mathcal{D}(F) \cap \mathcal{L}^{2}(\Omega) \rightarrow \mathcal{L}^{2}(\Omega),
$$

i.e.Г $\mathcal{X}=\mathcal{Y}=\mathcal{L}^{2}(\Omega)$. Here as before $\Gamma$

$$
\mathcal{D}(F)=\left\{a \in \mathcal{L}^{\infty}(\Omega) \mid \text { ess inf } a>0\right\} .
$$

To take this positivity constraint into account the Levenberg-Marquard method is modified in the following way: whenever the value of some iterate $a_{n}$ at some grid point happens to be negative this value is replaced by one. In all experiments with the Levenberg-Marquardt method $a_{0} \equiv 20$ is used as initial guess. The parameters $\rho$ and $\tau$ in (2.2) and (2.8) are freezed throughout to be $\rho=0.5$ and $\tau=2.5$.

The numerical results are summarized under three aspects: the regularizing properties of the iteration $\Gamma$ the performance with distributed data and with discrete measurementsTand the efficiency of the method as compared to a conventional LevenbergMarquardt strategy as described Te.g. Tin [6].

4.1. Regularizing properties. To verify the regularizing properties as predicted by Theorem 2.3 distributed data with two different signal to noise ratios (SNR) of 100 and 1000 (with respect to $\mathcal{L}^{2}(\Omega)$ ) have been used as input for the LevenbergMarquardt scheme. The reconstructions are shown in Figure 4.2 in form of a contour plot $\Gamma$ the level lines corresponding to multiples of ten. For an alternative visualization of these results consider Figure 4.3: it shows the traces of the phantom and of the two reconstructions along the dashed lines in Figure 4.2 connecting the points 


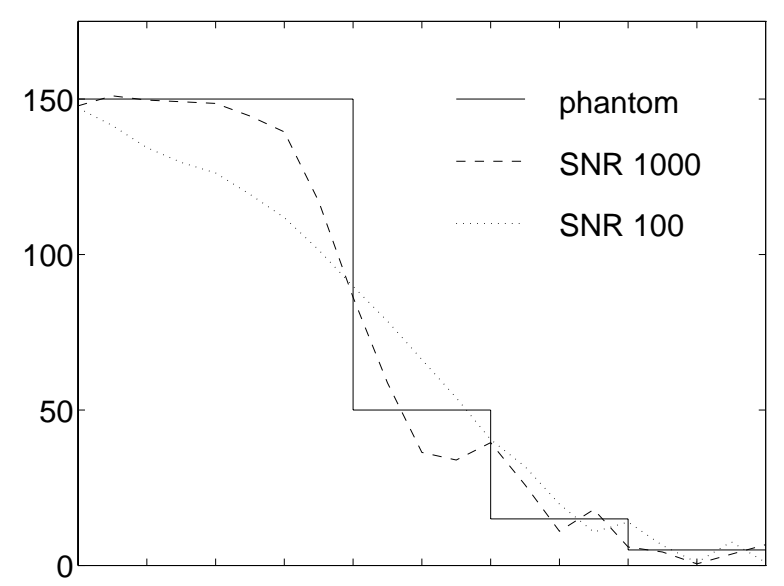

FIG. 4.3. Diagonal traces of the reconstructions
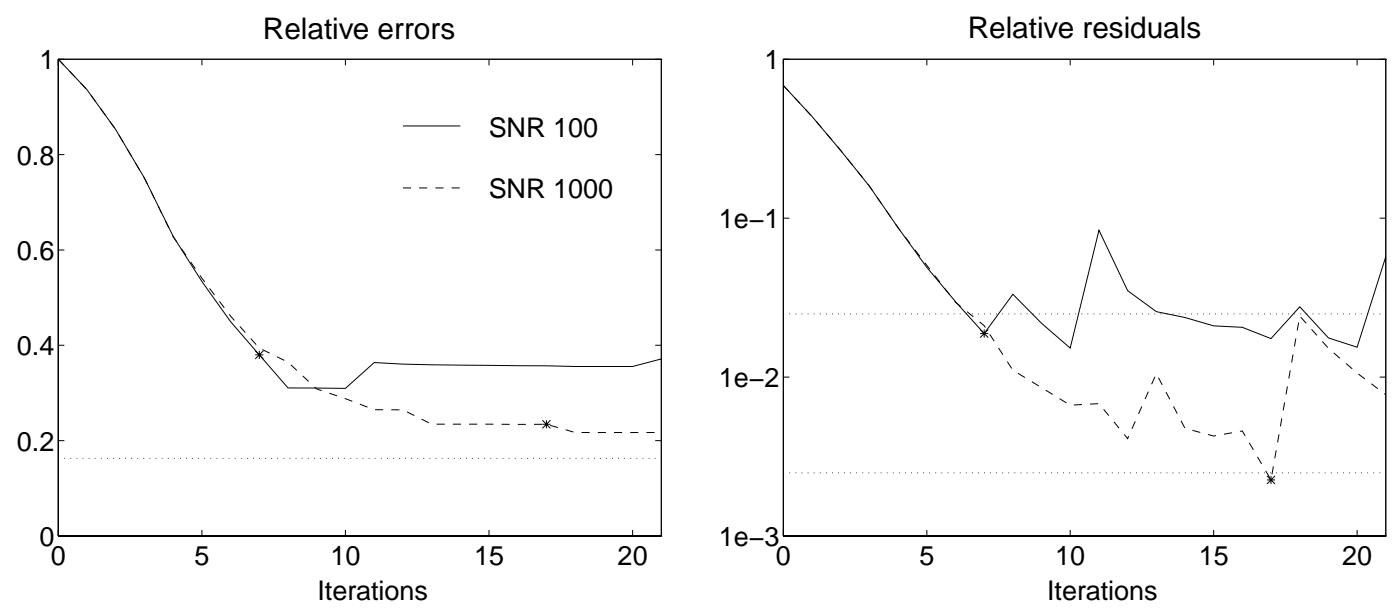

FIG. 4.4. Error and residual history for both noise levels

$(\xi, \eta)=(1,0)$ and $(6,5)$ (compare also Figure 4.1). The improvement with less noise is obvious from this plot.

It is even more instructive to look at the iteration history of the relative errors $\Gamma$ $\left\|a_{n}-a^{\dagger}\right\| /\left\|a^{\dagger}\right\|$ Tand of the relative residuals $\Gamma\left\|u^{\delta}-F\left(a_{n}\right)\right\| /\left\|u^{\delta}\right\|$. These histories are displayed in Figure 4.4. On each of these curvesTa star denotes the point where the algorithm is terminated according to the discrepancy principle (2.8): these are the points when the residual norm drops below $\tau$ /SNR with $\tau=2.5$ (as indicated by the dotted lines in the right-hand side plot). As the error history shows $\mathrm{in}$ both cases this termination point is close to optimal. Note that the dotted line indicates the error of the best approximation of $a^{\dagger}$ from the trial space of piecewise linears: the reconstructions of the algorithm are only worse by a factor of $2.4(\mathrm{SNR}=100)$ and 1.5 $(\mathrm{SNR}=1000)$ Trespectively.

Concerning the analysis of Sect. 2 the essentially monotonic decay of the iteration error is in nice agreement with the theory. In the case of SNR $=100$ the semiconvergent behaviour of the iteration is also obvious: without an adequate stopping criterion the iteration would eventually diverge. A similar phenomenon occurs in the case of less noise after some twenty more iterations. Finally concerning Theorem 2.4 Tnote that 
the residual does indeed decay linearly with a reduction of about $60 \%$ per step in the beginning of the iteration (the residuals are displayed in a semilogarithmic plot) but in the case of less noise this behaviour seems to deteriorate before the stopping point is reached. This may indicate that the necessary assumption (1.5) does not hold throughout.

4.2. The case of discrete measurements. In practice head data will only be measured at a finite number of wells within $\Omega$. Carrera and Neuman suggest to take data at the eighteen locations indicated by circles in Figure 4.1. The results presented in this subsection are based on point evaluations of the noise-corrupted piezometric head $u^{\delta}(\mathrm{SNR}=100)$ at these locations.

There are two principally different approaches in dealing with this case of discrete measurements. One option is to interpolate the eighteen measurements to obtain "distributed" data which can then be used in precisely the same way as above. If this is done the interpolated head function $\tilde{u}$ has a relative error of $\|\tilde{u}-u\| /\|u\| \approx 0.02$ (the corresponding signal to noise ratio is SNR $\approx 48.7$ ) Twhich corresponds to twice as much noise as for the respective "input function" $u^{\delta}$. It therefore comes as no surprise that the reconstructions of the diffusivity coefficient are somewhat worse: the best approximations are obtained after about ten iterations with a relative error somewhat below 0.39 (for comparison: the best error for SNR $=100$ has been $0.31 \Gamma c f$. Figure 4.4).

Alternatively one could consider the nonlinear operator $F$ as a mapping from $\mathcal{D}(F) \cap \mathcal{L}^{2}(\Omega)$ to $\mathbb{R}^{18}$ Ti.e. Tthe set of discrete data vectors equipped with the Euclidean norm. Of course the same Levenberg-Marquardt algorithm can be applied to this semidiscrete setting. With this approach the optimal reconstruction had an error of 0.38 Tbut it took more than fifty iterations to get there.

Note that the costs per iteration are not much different in either approach because the same number of boundary value problems (3.7) must be solved to evaluate $F^{\prime}(a)$. Therefore $i n$ this exampleTthe semidiscrete case turned out to be considerably more expensive without giving any better results.

4.3. A classical Levenberg-Marquardt implementation. In standard software packages the Lagrange parameter $\alpha_{n}$ of the Levenberg-Marquardt method is selected on the grounds of a trust region strategyГcf. [6Г15]. Let

$$
\|h\| \leq \eta_{n}
$$

define the trust region after $n$ iterations. In the $(n+1)$ st iteration the new approximation is defined as $a_{n+1}=a_{n}+h_{n}$ where $h_{n}$ is the minimizer of

$$
\left\|u^{\delta}-F\left(a_{n}\right)-F^{\prime}\left(a_{n}\right) h\right\|^{2} \longrightarrow \min .
$$

subject to the constraint (4.1). Afterwards the radius $\eta_{n}$ of the trust region is modified according to the improvement of the nonlinear objective function $\left\|u^{\delta}-F(a)\right\|$ during this iteration.

To compare the new Levenberg-Marquardt scheme with those more standard ones the following trust region strategy from [6Гp. 145] has been implemented. Denote by

$$
\Delta f:=\left\|u^{\delta}-F\left(a_{n}\right)\right\|-\left\|u^{\delta}-F\left(a_{n}+h\right)\right\|
$$

the actual reduction of the objective function $\Gamma$ and compare $\Delta f$ with the reduction

$$
\Delta f_{\text {pred }}:=\left\|u^{\delta}-F\left(a_{n}\right)\right\|-\left\|u^{\delta}-F\left(a_{n}\right)-F^{\prime}\left(a_{n}\right) h\right\|
$$



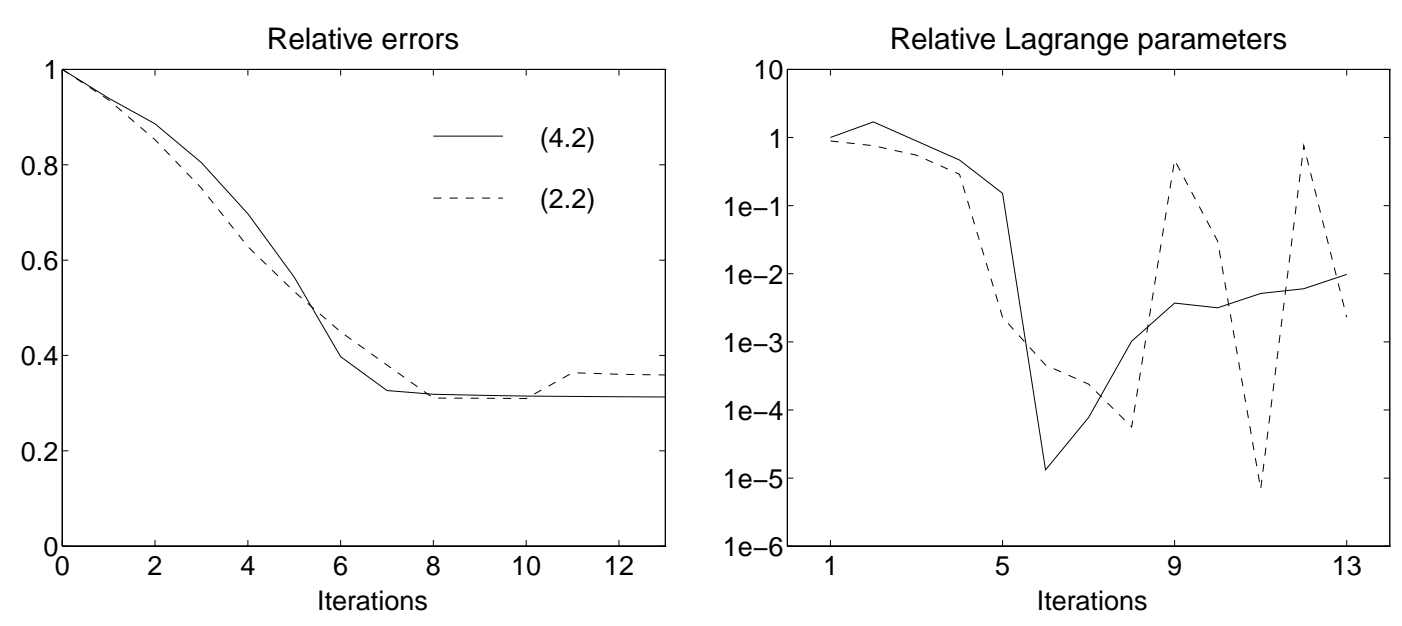

FIG. 4.5. Error history and $\alpha$-history for both implementations (SNR $=100)$

predicted by the linear model. Then the trust region radius $\eta_{n}$ is updated in the following way:

$$
\eta_{n+1}=\left\{\begin{array}{cl}
\sqrt{2} \eta_{n} & \Delta f \geq 0.75 \Delta f_{\text {pred }} \\
\eta_{n} / \sqrt{2} & \Delta f \leq 0.1 \Delta f_{\text {pred }} \\
\eta_{n} & \text { else. }
\end{array}\right.
$$

No line search is implemented in this scheme but for a robust performance of this algorithm it is essential to discard updates $h_{n}$ for which $\Delta f$ is negative $\mathrm{i}$ i.e. $\Gamma$ for which the objective function increases; such iterations are said to be unsuccessful. Unsuccessful iterations are repeated with a reduced trust region radius $\eta_{n} \leftarrow \eta_{n} / 2$ until they eventually become successful. (In this implementation no Armijo-type condition $\Gamma$ cf. [15Г p. 315] Tis imposed on an iteration to be successful). Note that unsuccessful iterations do not show up in the iteration history plots in Figure 4.5T but they are nevertheless essential for a comparison of the two algorithms because unsuccessful iterations are as expensive as successful ones.

The two Levenberg-Marquardt implementations (the new scheme with $\alpha_{n}$ chosen from (2.2) and the classical one with the trust region strategy (4.2)) have both been tested on the model problem with $\mathrm{SNR}=100$. Consider Figure 4.5 for the iteration histories: the solid lines refer to the conventional implementation using (4.2) whereas the dashed lines correspond to the new algorithm. As can be seen from the error history in the left-hand plot the performance of the two methods is quite comparable in the first ten iterations. In both cases the error is reduced to about 0.31 after eight iterations which is essentially optimal. But not only the iteration history is comparable up to this point; the reconstructions are similar too $\Gamma$ which is quite obvious from Figure 4.6 which shows the traces of the two reconstructions along the dashed line in Figure 4.1.

After seven iterations ThoweverTevery further iteration of the conventional implementation happens to run into two unsuccessful iterations in the average to a significant computational overhead. In fact Tthe algorithm would diverge if unsuccessful iterations were considered successful. (Note that the new Levenberg-Marquardt scheme does not distinguish between successful and unsuccessful iterations). The impact on the chosen Lagrange parameters can be seen from the right-hand plot of 


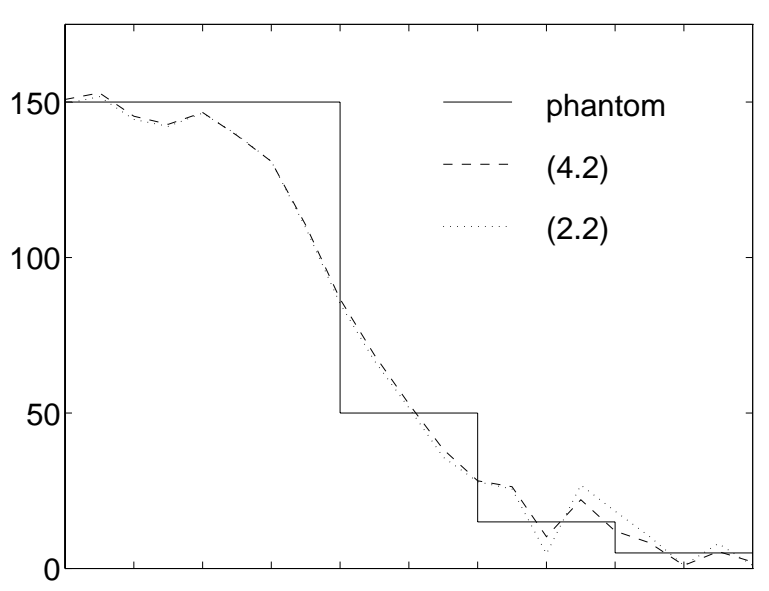

FIG. 4.6. Reconstructions (trace) after eight iterations

Figure 4.5. It shows the "relative Lagrange parameters" for the successful iterations $\Gamma$ i.e. $\Gamma \alpha_{n} /\left\|F^{\prime}\left(a_{n}\right)\right\|^{2}$ Tthe Lagrange parameter divided by the squared norm of the linear operator.

It seems as if the general tendency in choosing the parameter $\alpha_{n}$ from the two adaptive strategies (2.2) and (4.2) is not much different - at least in the convergent stage of the iteration. Both schemes first reduce the parameter subsequently down to a (relative) value between $10^{-4}$ and $10^{-5}$. This value is then recognized as being too small $\Gamma$ and hence increased. While the new strategy of changing $\alpha_{n}$ turns out to be quite flexible The conventional implementation is somewhat more lethargic; this could of course be modified by replacing $\sqrt{2}$ by a somewhat larger factor in (4.2).

In summaryTthe standard Levenberg-Marquardt method seems to give the same accuracy as the new scheme for which regularizing properties can be established. On the other hand $\mathrm{T}$ it is not clear what kind of stopping rule would be appropriate for the standard implementation. Although this may not be such a delicate question as far as stability is concerned $\mathrm{Tit}$ is essential for the computational expenses: if only ten instead of eight iterations were performed with the present trust region implementation this would result in additional seven unsuccessful iterations $\Gamma$ and hence $\Gamma$ the standard implementation would require about twice as much work as the new scheme.

5. Conclusion. The adaptive strategy (2.2) for choosing the Lagrange parameter in the Levenberg-Marquardt method has been shown to be a stable method. In combination with the discrepancy principle (2.8) as a stopping rule this algorithm is a regularization method in the sense of [7ГDef. 3.1] for (at least some) ill-posed problems. In particularTthis method applies to the reconstruction of diffusivity parameters in elliptic and parabolic equations from distributed data. Problems of this type arise $\Gamma$ e.g. Tin groundwater hydrology.

The numerical experiments seem to confirm the theoretical results. It turns out $\Gamma$ however $\Gamma$ that a conventional trust region strategy seems to give comparable reconstructions $\mathrm{T}$ at least for the particular example considered in Sect. 4. Whether such trust region implementations of the Levenberg-Marquardt iteration are also regularization methods in the aforementioned sense remains a very interesting open problem. Especially $\mathrm{T}$ this concerns the design of suitable stopping criteria for these implemen- 
tations. The numerical examples indicate that the same stopping rule (2.8) Ti.e.T the discrepancy principle $\Gamma$ might be a worthwhile candidate.

\section{REFERENCES}

[1] R. A. Adams, Sobolev Spaces, Academic Press, New York, San Francisco, London, 1975.

[2] H. T. Banks AND K. Kunisch, Estimation Techniques for Distributed Parameter Systems, Birkhäuser, Basel, Boston, Berlin, 1989.

[3] J. CARRERA AND S. P. NeUman, Estimation of aquifer parameters under transient and steady state conditions: 3. Applications to synthetic and field data, Water Resour. Res., 22 (1986), pp. $228-242$.

[4] F. COLONIUS AND K. KUNISCH, Output least squares stability in elliptic systems, Appl. Math. Optim., 19 (1989), pp. 33-63.

[5] R. Dautray and J.-L. Lions, Mathematical Analysis and Numerical Methods for Science and Technology, Vol. 5: Evolution Problems I, Springer, Berlin, Heidelberg, New York, 1992.

[6] J. E. Dennis AND R. B. SchnaBeL, Numerical Methods for Unconstrained Optimization and Nonlinear Equations, SIAM, Philadelphia, 1996.

[7] H. W. Engl, M. Hanke, and A. NeubaueR, Regularization of Inverse Problems, Kluwer Academic Publ., Dordrecht, 1996.

[8] H. W. Engl AND W. Rundell, eds., Inverse Problems in Diffusion Processes, SIAM, Philadelphia, 1995.

[9] P. Grisvard, Elliptic Problems in Nonsmooth Domains, Pitman, Boston, London, Melbourne, 1985.

[10] C. W. Groetsch, The Theory of Tikhonov Regularization for Fredholm Equations of the First Kind, Pitman, Boston, London, Melbourne, 1984.

[11] M. HANKE, Regularizing properties of a truncated Newton-CG algorithm for nonlinear inverse problems, submitted.

[12] K. ITO AND K. KUNISCH, On the injectivity and linearization of the coefficient-to-solution mapping for elliptic boundary value problems, J. Math. Anal. Appl., 188 (1994), pp. 1040-1066.

[13] C. KRAVARIS AND J. H. SEINFELD, Identification of parameters in distributed parameter systems by regularization, SIAM J. Control Optim., 23 (1985), pp. 217-241.

[14] O. A. LADYZHENSKAYA AND N. N. URAL'TSEVA, Linear and Quasilinear Elliptic Equations, Academic Press, New York, London, 1968.

[15] S. G. NASH AND A. Sofer, Linear and Nonlinear Programming, McGraw-Hill, New York, 1996.

[16] C. R. VoGEL, An overview of numerical methods for nonlinear ill-posed problems, in Inverse and Ill-Posed Problems, H. W. Engl and C. W. Groetsch, eds., Orlando, 1987, Academic Press, pp. 231-245.

[17] W. W.-G. YEH, Review of parameter identification procedures in groundwater hydrology: the inverse problem, Water Resour. Res., 22 (1986), pp. 95-108. 